\title{
A Novel Insertion Mutation on Exon 20 of Epidermal Growth Factor Receptor, Conferring Resistance to Erlotinib
}

\author{
Nawazish A. Khan ${ }^{a} \quad$ Saied Mirshahidi ${ }^{b} \quad$ Hamid R. Mirshahidi ${ }^{a}$ \\ a Loma Linda University Cancer Center, and ${ }^{b}$ Biospecimen Laboratory, Loma Linda \\ University Cancer Center, Loma Linda, Calif., USA
}

\section{Key Words}

Epidermal growth factor receptor - Tyrosine kinase receptor inhibitors · Mutations · Exon 20 . Non-small cell lung cancer · Erlotinib

\begin{abstract}
The epidermal growth factor receptor (EGFR) is a transmembrane glycoprotein tyrosine kinase receptor. The small-molecule tyrosine kinase receptor inhibitors (TKIs) are in clinical use to treat non-small cell lung cancer with EGFR mutations. Variable tumor responses to erlotinib and gefitinib have been observed. The response to these TKIs varies by the type of EGFR mutations found in the tumor. The deletion on exon 19 and the L858R substitution on exon 21 constitute the most frequent mutations and are known to show good response to TKIs. However, mutations on exon 20 are less common and seem to respond poorly to TKIs. In clinical settings, the reported response of exon 20 mutations to reversible TKIs (both gefitinib and erlotinib) remains inconstant. The type of coexisting mutation seems to affect the response of these insertions to TKIs. We herein present a case of disease progression despite the use of erlotinib in a female patient who had a novel insertion mutation on exon 20. Our patient was a never-smoker and was identified to have a Pro772_His773insGInCysPro mutation on exon 20. She had previously been treated with cisplatin and gemcitabine and then with carboplatin and pemetrexed. She was treated with erlotinib upon intolerance to second-line chemotherapy and did not respond. Our patient had a novel insertion mutation on exon 20, which was found to be resistant to erlotinib.


Khan et al.: A Novel Insertion Mutation on Exon 20 of Epidermal Growth Factor Receptor, Conferring Resistance to Erlotinib

\section{Background}

The epidermal growth factor receptor (EGFR) is a transmembrane glycoprotein that constitutes one of the four members of the ErbB family of tyrosine kinase receptors [1]. The small-molecule tyrosine kinase receptor inhibitors (TKIs) are in clinical use to treat nonsmall cell lung cancer (NSCLC). The two TKIs used at present are erlotinib and gefitinib, usually employed in selected patients with NSCLC and showing variable responses. [2]. The response to these TKIs varies by the type of EGFR mutations found in the tumor. These mutations exist on exons 18-21 and cluster on chromosome 7p [3]. The deletion on exon 19 and the L858R substitution on exon 21 constitute the most frequent mutations. Tumors expressing these two mutations are known to show good response to TKIs.

The other, less common mutations include insertions or in-frame duplications on exon 20. These mutations seem to have poor response to TKIs [4]. The significance of this resistance conferred by different insertion mutations on exon 20 is not well established. The use of TKIs against tumors expressing these insertion mutations is not contraindicated. We herein present a case of disease progression despite the use of erlotinib in a patient who expressed a novel insertion mutation on exon 20. Written informed consent was obtained from the patient for the publication of this case report and any accompanying images. A copy of the written consent is available for review from the editor in chief of this journal.

\section{Case Presentation}

The patient was a 74-year-old, non-smoking female. She was diagnosed with stage IIb NSCLC (adenocarcinoma) and underwent lobectomy in 2009. She received four cycles of cisplatin and gemcitabine as adjuvant therapy. Her disease remained stable for more than 3 years. Later, positron emission tomography (PET) scanning revealed disease progression. The CT-guided biopsy of the spinal mass confirmed metastatic disease consistent with adenocarcinoma of the lung. Biopsy material from the bone lesion was sent to Genzyme Genetics Laboratories to test the EGFR mutation and anaplastic lymphoma kinase (ALK) translocation. The laboratory was unable to process the tests due to decalcification of the bone. The patient received radiation therapy of the spinal lesions. In addition, she was started on carboplatin and pemetrexed, and she received proton radiation therapy of the mediastinum. Chemotherapy was discontinued after five cycles (September 26, 2011December 12, 2011) due to thrombocytopenia. Her disease remained stable with this regimen for about 6 months. Later, however, repeat imaging studies showed that she had disease progression. We decided that we would use TKIs if the patient displayed an EGFR mutation. Since the laboratory was unable to process for EGFR mutations on the metastatic bone lesion, the sample from the earlier lobectomy was sent for testing for EGFR mutation and ALK translocation status. The laboratory results showed that the rearrangement involving the ALK gene was negative, and the patient had a Pro772_His773insGlnCysPro mutation on exon 20.

Since there is no confirmatory data on the TKI response of this unique type of EGFR mutation, we decided to use erlotinib. The patient's Eastern Cooperative Oncology Group (ECOG) performance status was 1 at the beginning of the treatment. The pretreatment PET scan study was obtained (fig. 1). She was started on $150 \mathrm{mg}$ of oral erlotinib (Tarceva) daily and tolerated it well. She experienced a minimal facial rash and diarrhea during the therapy. The biomarkers and repeat imaging revealed disease progression after 6 weeks of treatment 
Khan et al.: A Novel Insertion Mutation on Exon 20 of Epidermal Growth Factor Receptor, Conferring Resistance to Erlotinib

with erlotinib. Her ECOG performance status declined to 2, most likely due to disease progression, as evident on the posttreatment PET scan (fig. 2).

\section{Discussion}

EGFR is part of the ErbB family of cell surface receptor tyrosine kinases. These receptors are involved in signal transduction pathways that regulate proliferation and apoptosis. Exon 20 of EGFR encompasses nucleotides that translate into amino acid positions 762-823. The C-helix facilitates the correct orientation of amino acids and ends at Met766. The preferential location of the majority of the insertion mutations is following the C-helix. EGFR expresses a broad spectrum of somatic mutations. The insertion mutations on exon 20 were found to be mutually exclusive with other genetic alterations in NSCLC except PIK3CA. These insertions vary from three to twelve base pairs and account for 11\% of all EGFR mutations. Patients who never smoked were found to have the highest incidence; sex, ethnicity and stage at diagnosis have not shown any correlations. The insertion mutations on exon 20 seem to have poor response to TKIs. The variable response to TKIs reaffirms the heterogeneity of this receptor $[5,6]$.

Preclinical data suggest less favorable responses to reversible TKIs (gefitinib and erlotinib). These exon 20 insertions were also found to be far less sensitive to irreversible TKIs (neratinib and afatinib) when compared to L858R and exon 19 mutations [5]. In clinical settings, the reported response to reversible TKIs (both gefitinib and erlotinib) remains inconstant. In the Taiwanese population, the treatment response to gefitinib was found to be $25 \%$, which was much lower when compared to exon 19 and L858R mutations. The majority of these patients also had other coexisting EGFR mutations. The coexisting point mutations on exon 20 that rendered the patients resistant to gefitinib were G719A, V769L, W731 Stop, L858R, L 861Q and delE749_T751insVA [7]. The type of coexisting mutation seems to affect the response of these insertions to TKIs.

The exon 20 mutation (S768I+G719X) seemed to show some response to gefitinib while it coexisted with a mutation on exon 18 rather than as a single mutation [8]. The response of malignant pleural effusions to gefitinib has also been reported in NSCLC patients with EGFR mutations. The patients carrying mutations, including those with a deletion on exon 19, missense on exon 21 and a mutation on exon 18, responded to gefitinib. However, a patient carrying an in-frame insertion mutation on exon 20 (P772_H773insTyrAsnPro+H773Y) was found to have progression of pleural effusion while on gefitinib [9]. In contrast, another patient carrying an in-frame insertion (S768) on exon 20 of his tumor was reported to show response to gefitinib and was found to have stable disease so that gefitinib was used as a second-line therapy [10].

Our patient was a never-smoker and was identified to have a Pro772_His773insGlnCysPro mutation on exon 20. This mutation at amino acid 772 has not been reported in the literature $[5,7,9,11,12]$ (table 1 ). These insertion mutations on this amino acid account for $17 \%$ of all insertion mutations on exon 20 . These mutations exist on the loop following the C-helix [5]. Another, recently reported insertion mutation, P772_H773insVal, is characterized by an insertion of a nucleotide triplet GTT. This patient was not offered a TKI and was treated with six courses of cisplatin/pemetrexed chemotherapy and had disease progression. The patient was reported to have disease stabilization on second-line chemotherapy with docetaxel [12].

The response to TKIs on exon 20 insertion-mutated NSCLCs was studied in eight different representative mutations of exon 20 in vitro. The insertion mutations located in the C- 
Khan et al.: A Novel Insertion Mutation on Exon 20 of Epidermal Growth Factor Receptor, Conferring Resistance to Erlotinib

helix showed response to TKI when compared to the ones located in the loop following the C-helix. The A763_Y764insPheGlnGluAla mutation located in the C-helix showed response to erlotinib or other TKIs [13].

The novel insertion mutation Pro772_His773insGlnCysPro, which was identified in our patient, is most likely located in the loop following the C-helix. The patient did not respond to erlotinib and had disease progression. This lack of response confirms the resistance of this particular mutation to TKIs. As reported earlier, these insertion mutations seem to confer resistance to TKIs even when they coexist with other mutations [7]. The recent preclinical data reported by Yasuda et al. [13] reaffirm the poor response to TKIs for the mutations that exist on this particular loop of exon 20.

\section{Acknowledgement}

The division of Hematology/Oncology at Loma Linda University, Loma Linda, Calif., USA, supported the writing of the manuscript and paid processing fees.

\section{Disclosure Statement}

The authors have no relevant conflicts of interest to declare.

\section{References}

1 Herbst S: Review of epidermal growth factor receptor biology. Int J Radiat Oncol Biol Phys 2004;59(suppl 2):21-26.

-2 Jänne PA, Gurubhagavatula S, Yeap BY, Lucca J, Ostler P, Skarin AT, Fidias P, Lynch TJ, Johnson BE: Outcomes of patients with advanced non-small cell lung cancer treated with gefitinib (ZD1839, 'Iressa') on an expanded access study. Lung Cancer 2004;44:221-230.

-3 Yuan S, Yu SL, Chen HY, Hsu YC, Su KY, Chen HW, Chen CY, Yu CJ, Shih JY, Chang YL, Cheng CL, Hsu CP, Hsia JY, Lin CY, Wu G, Liu CH, Wang CD, Yang KC, Chen YW, Lai YL, Hsu CC, Lin TC, Yang TY, Chen KC, Hsu KH, Chen JJ, Chang GC, Li KC, Yang PC: Clustered genomic alterations in chromosome 7p dictate outcomes and targeted treatment responses of lung adenocarcinoma with EGFR-activating mutations. J Clin Oncol 2011;29:3435-3442.

4 Wu JY, Yu CJ, Chang YC, Yang CH, Shih JY, Yang PC: Effectiveness of tyrosine kinase inhibitors on 'uncom mon' epidermal growth factor receptor mutations of unknown clinical significance in non-small cell lung cancer. Clin Cancer Res 2011;17:3812-3821.

5 Yasuda H, Kobayash Si, Costa DB: EGFR exon 20 insertion mutations in non-small-cell lung cancer: preclinical data and clinical implications. Lancet Oncol 2012;13:e23-e31.

-6 Arcila ME, Nafa K, Chaft JE, Rekhtman N, Lau C, Reva BA, Zakowski MF, Kris MG, Ladanyi M: EGFR exon 20 insertion mutations in lung adenocarcinomas: prevalence, molecular heterogeneity, and clinicopathologic characteristics. Mol Cancer Ther 2013;12:220-229.

7 Wu JY, Wu SG, Yang CH, Gow CH, Chang YL, Yu CJ, Shih JY, Yang PC: Lung cancer with epidermal growth factor receptor exon 20 mutations is associated with poor gefitinib treatment response. Clin Cancer Res 2008;14:4877-4882.

8 Lund-Iversen M, Kleinberg L, Fjellbirkeland L, Helland Å, Brustugun OT: Clinicopathological characteristics of 11 NSCLC patients with EGFR-exon 20 mutations. J Thorac Oncol 2012;7:1471-1473.

9 Chen CH, Gow CH, Yu CJ, Shih JY, Hsu YC, Lee WY, Yang PC, Kuo SH: Clinical response of gefitinib on malignant pleural effusions in patients with non-small cell lung cancer. J Cancer Mol 2008;4:23-28.

10 Masago K, Fujita S, Irisa K, Kim YH, Ichikawa M, Mio T, Mishima M: Good clinical response to gefitinib in a non-small cell lung cancer patient harboring a rare somatic epidermal growth factor gene point mutation; codon 768 AGC > ATC in exon 20 (S768I). Jpn J Clin Oncol 2010;40:1105-1109.

-11 Sasaki H, Endo K, Takada M, Kawahara M, Kitahara N, Tanaka H, Okumura M, Matsumura A, Iuchi K, Kawaguchi T, Kawano O, Yukiue H, Yokoyama T, Yano M, Fujii Y: EGFR exon 20 insertion mutation in Japanese lung cancer. Lung Cancer 2007;58:324-328. 


\section{Case Reports in Oncology}

\begin{tabular}{l|l}
\hline Case Rep Oncol 2014;7:491-496 \\
\hline DOI: 10.1159/000365325 & $\begin{array}{l}\text { C 2014 S. Karger AG, Basel } \\
\text { www.karger.com/cro }\end{array}$ \\
\hline
\end{tabular}

Khan et al.: A Novel Insertion Mutation on Exon 20 of Epidermal Growth Factor Receptor, Conferring Resistance to Erlotinib

12 Zupa A, Vita G, Landriscina M, Possidente L, Aieta M, Tartarone A, Improta G: Identification of a new insertion in exon 20 of EGFR in a woman with NSCLC. Med Oncol 2012;29:3198-201.

13 Yasuda H, Sng NJ, Yeo WL, Figueiredo-Pontes LL, Kobayashi S, Costa DB: Sensitivity of EGFR exon 20 insertion mutations to EGFR inhibitors is determined by their location within the tyrosine kinase domain of EGFR (abstract 23). Cancer Res 2012;72:23.

Table 1. Reported EGFR mutations on exon 20 at amino acid position 772

\begin{tabular}{lll}
\hline $\begin{array}{l}\text { Amino acid } \\
\text { position }\end{array}$ & EGFR mutation & References \\
\hline 772 & & \\
& Pro772His773insProArg & {$[5,7,9,11,12]$} \\
& Pro772His773insTyrAsnPro & \\
& Pro772His773insX & \\
& Pro772His773insAspProHis & \\
& Pro772His773insAspAsnPro & \\
& Pro772His773insGlnVal & \\
& Pro772His773insThrProHis & \\
& Pro772His773insAsn & \\
& Pro772His773insVal & \\
& Pro772His773insGlnCysPro* \\
\hline
\end{tabular}

* Mutation detected in our patient.
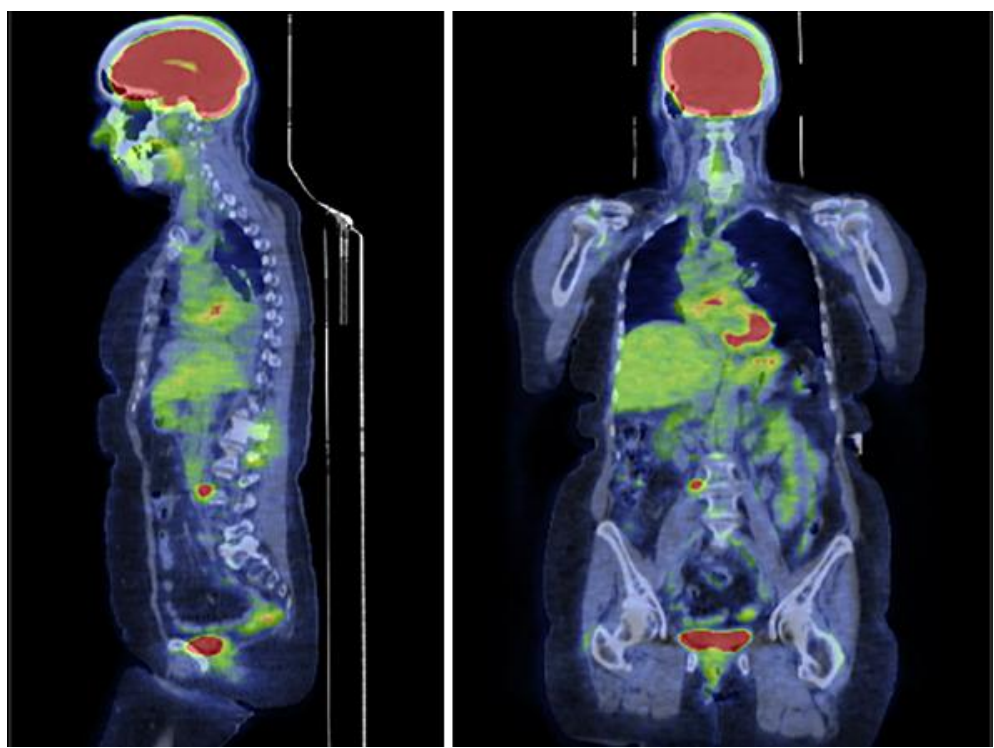

Fig. 1. Pretreatment PET scan. 6.7 SUV of the retroperitoneal right periaortic lymph node measures $2.2 \times$ $1.3 \mathrm{~cm}$. Increased activity of $3.8 \mathrm{SUV}$ in the spinous process of L3. Focal uptake of $4.5 \mathrm{SUV}$ seen in the posterior left ilium. 


\section{Case Reports in Oncology}

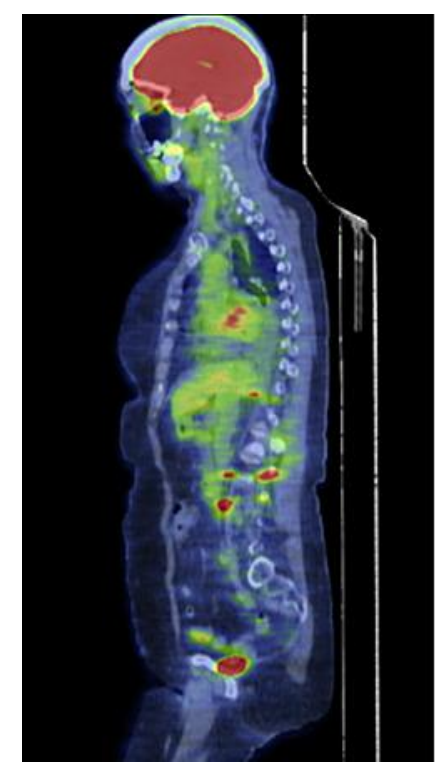

\begin{tabular}{l|l}
\hline Case Rep Oncol 2014;7:491-496 \\
\hline DOI: $10.1159 / 000365325$ & $\begin{array}{l}\text { C 2014 S. Karger AG, Basel } \\
\text { www.karger.com/cro }\end{array}$ \\
\hline
\end{tabular}

Khan et al.: A Novel Insertion Mutation on Exon 20 of Epidermal Growth Factor Receptor, Conferring Resistance to Erlotinib

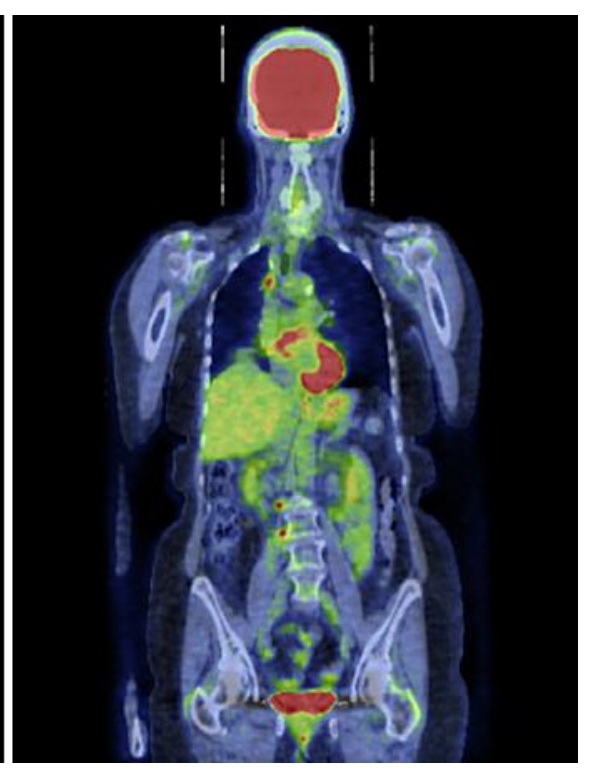

Fig. 2. Post-treatment PET scan. Mildly increased FDG uptake in the right hilar region, metastatic lymphadenopathy not excluded. New focal increased uptake in the right pedicle of L3 with a maximum SUV of 5. The more extensive FDG uptake seen in the posterior left ilium adjacent to the sacroiliac joint, measuring up to $8.8 \mathrm{SUV}$, corresponds to patchy sclerosis. Interval increase in the hypermetabolic osseous lesions as well as hypermetabolic retroperitoneal, retrocrural and mediastinal adenopathy, as detailed above, compatible with the progression of metastasis (seen in a different image not shown here). 